Rev. Adv. Mater. Sci. 57 (2018) 72-81

\title{
STRUCTURAL CHARACTERIZATION OF MOVPE GROWN AIGaN/GaN FOR HEMT FORMATION
}

\author{
V.N. Popok', T.S. Aunsborg', R.H. Godiksen', P.K. Kristensen'1, R.R. Juluri², \\ P. Caban ${ }^{3}$ and K. Pedersen ${ }^{1}$
}

\author{
'Department of Materials and Production, Aalborg University, Skjernvej 4A, 9220 Aalborg, Denmark \\ ${ }^{2}$ Interdisciplinary Nanoscience Center (iNANO), Aarhus University, \\ Gustav Wieds Vej 14, 8000 Aarhus C, Denmark \\ ${ }^{3}$ Institute of Electronic Materials Technology, Wolczynska 133, 01-919 Warsaw, Poland
}

Received: February 02, 2018

\begin{abstract}
Results on structural, compositional, optical and electrical characterization of MOVPE grown AIGaN/GaN heterostructures with focus on understanding how the AIN buffer synthesis affects the top films are reported. The study demonstrates very good correlation between different methods providing a platform for reliable estimation of crystalline quality of the AIGaN/GaN structures and related to that electrical performance which is found to be significantly affected by threading dislocations (TD): higher TD density reduces the electron mobility while the charge carrier concentration is found to be largely unchanged. The attempt to vary the ammonia flow during the AIN synthesis is found not to affect the film composition and dislocation densities in the following heterostructures. An unusual phenomenon of considerable diffusion of $\mathrm{Ga}$ from the GaN film into the AIN buffer is found in all samples under the study. The obtained results are an important step in optimization of AIGaN/GaN growth towards the formation of good quality HEMT structures on sapphire and transfer of technology to Si substrates by providing clear understanding of the role of synthesis parameter on structure and composition of the films.
\end{abstract}

\section{INTRODUCTION}

AlGaN/GaN heterostructures are of high research and industrial interest for the production of high electron mobility transistors (HEMT) utilizing the twodimensional electron gas (2DEG) induced at the interface due to polarization effect [1]. Superior properties of $\mathrm{GaN}$ as a wide band gap semiconductor material and the 2DEG formation are advantageous for device implementation in power switching applications [2]. Since GaN bulk single crystals are very expensive and hardly commercially available, technological development of $\mathrm{GaN}$-based devices relies on heteroepitaxy of films. One of the most widely used approaches is metal organic chemical vapour deposition also called metal organic vapor phase epitaxy (MOVPE) [3]. Typically sapphire wafers are used as substrates. The choice of sapphire is related to low cost and acceptable difference of thermal expansion coefficient with respect to $\mathrm{GaN}[4,5]$. However, the strong industry interest in mass production of $\mathrm{GaN}$ devices requires transfer of III-N epitaxy to $\mathrm{Si}[4,6-9]$. One of the main problems of heteroepitaxy is poor matching of lattice parameters of GaN to the most widely used substrates such as sapphire and $\mathrm{Si}$. The epitaxial growth generates defects among which threading dislocations (TD) are the most prominent ones reaching very high densities of $10^{9}-10^{10} \mathrm{~cm}^{-2}$. This seriously affects performance of the devices, in particular, it leads to increased leakage currents, lowering of electron mobility etc. [10]. To improve crystalline quality of GaN and accommodate the stresses (and thus reduce TD density) a nucleation layer (typically AIN) and

Corresponding author: V.N. Popok, e-mail:vp@mp.aau.dk

(c) 2018 Advanced Study Center Co. Ltd. 
then a buffer layer (or a stack of AIGaN layers with gradually decreasing concentration of $\mathrm{Al}$ ) are synthesised. Although reducing the TD density to low $10^{8}$ or even approximately $5 \times 10^{7} \mathrm{~cm}^{-2}$ is found to be possible by optimisation of epitaxy parameters as shown elsewhere [6,11], it does not solve the problem completely.

Additionally to TDs, the formation of point defects and non-abrupt interfaces significantly affect the 2DEG by introducing scattering of charge carriers [12]. It is shown that growth of a thin AIN interlayer at the GaN/AIGaN interface helps to achieve sharper interfaces reducing the $G$ a and $A l$ inter-diffusion, thus, decreasing alloy scattering $[13,14]$. The abruptness of the top interface can be judged from the surface morphology of the as-grown films. A smooth stepped surface with low roughness would imply a layer-bylayer growth and sharp interfaces between the layers. Since atomic force microscopy (AFM) is one of the appropriate methods to study surface morphology, it can also be suggested as an indirect tool for estimation of interface abruptness in the very top layers of heterostructures. Point defects such as impurities and vacancies incorporated or formed during the growth act as traps for charge carriers. In devices, they can form pathways for parasitic currents lowering the performance of the device, e.g. by lowering the signal-to-noise ratio [15]. Such impurities are most conveniently studied optically. For example, the luminescence of several defect bands can be investigated in GaN at low temperatures.

Thus, a lot of research is currently carried out to optimize growth of III-N heterostructures, reduce dislocation density, other types of defects and imperfections with an aim to improve materials quality. Since the synthesis parameters are very much dependent on the reactor type, they need to be carefully adjusted and optimized for the given epitaxial system and synthesis of particular heterostructures.

In the current work we present results of structural, compositional, optical and electrical studies of $\mathrm{AIGaN} / \mathrm{GaN}$ heterostructures. In particular, it is investigated how the conditions of buffer AIN growth affect the quality of the top III-N layers and the formation of 2DEG. The obtained results are intended to optimize the MOVPE growth procedure towards formation of HEMT structures on sapphire and to be used in the following transfer of synthesis to $\mathrm{Si}$ substrates.

\section{EXPERIMENTAL}

AlGaN/GaN heterostructures are grown on 2-inch single side polished (0001)-oriented sapphire substrates with a maximum off-cut of $0.2^{\circ}$ to $\mathrm{m}$ plane. The synthesis is performed using an AIX 200/ 4 RF-S MOVPE low-pressure reactor utilizing source gases of trimethylaluminium (TMAl), trimethylgallium (TMGa), ammonia $\left(\mathrm{NH}_{3}\right)$, and silane $\left(\mathrm{SiH}_{4}\right)$ as well as hydrogen $\left(\mathrm{H}_{2}\right)$ as carrier gas.

In this work, four types of heterostuctures which can be used for HEMT formation are grown. For all samples, a thin AIN nucleation layer is synthesized at the same conditions (reactor pressure of $50 \mathrm{mbar}$, reactor temperature $T_{\mathrm{R}}=720^{\circ} \mathrm{C}$, flux ( $F$ ) of TMAl of $2.2 \mu \mathrm{mol} / \mathrm{min}$ and $\mathrm{NH}_{3}$ of $13.4 \mathrm{mmol} / \mathrm{min}$, baking time of 6 min with $T_{\mathrm{R}}$ ramping from 720 to $1150^{\circ} \mathrm{C}$ and then $2.5 \mathrm{~min}$ at constant temperature). The main difference between the four structures is in the growth of following AIN layer which is carried out at $T_{\mathrm{R}}=$ $1220^{\circ} \mathrm{C}$ constant $F_{\text {TMAI }}=21.8 \mu \mathrm{mol} / \mathrm{min}$ but various $F_{\mathrm{NH} 3}: 2.2 \mathrm{mmol} / \mathrm{min}$ for samples $A ; 5.6 \mathrm{mmol} / \mathrm{min}$ for samples B; $6.7 \mathrm{mmol} / \mathrm{min}$ for samples $C$ and linearly decreasing from 2.2 to $0.2 \mathrm{mmol} / \mathrm{min}$ for samples $D$. The change in ammonia flux leads to growth of AIN layers of different thickness [1]. Thus, samples $A$ and $D$ are expected to have thicker while samples $B$ and $C$ have thinner AIN buffer layers. This issue will be addressed in more detail in the Results and Discussion section. To synthesize GaN, TMGa with $F=31.1 \mu \mathrm{mol} / \mathrm{min}$ and $\mathrm{NH}_{3} F=89$ $\mu \mathrm{mol} / \mathrm{min}$ are supplied for 1 minute, then TMGa flux is increased to $88.6 \mu \mathrm{mol} / \mathrm{min}$ and kept at this value to the end of this growth stage for all samples. The reactor pressure for $\mathrm{GaN}$ growth is kept at $200 \mathrm{mbar}$. Finally, a nm thin AIN, three AIGaN barrier and capping GaN layers (see Fig. 1) are formed at pressure of 70 mbar in the same way for all heterostructures. Total thickness of the stack is grown to be about 25 $\mathrm{nm}$. To control the synthesis, in-situ reflectance measurements are performed using a LaytecEpiCurve TT system. Two samples of each type (with different AIN layers) are fabricated.

Composition, structure, surface morphology, optical and electrical properties of the samples are studied by several methods. AFM measurements are carried out in tapping mode using Ntegra-Aura nanolaboratory (from NT-MDT). Commercial silicon and $\mathrm{WCO}_{2}$-coated cantilevers are utilized. On the one hand, use of conductive cantilevers helps to minimize effects of charging and polarization which often occurs on GaN surfaces. On the other hand, the larger curvature radius of coated tips (ca. 35 $\mathrm{nm})$ compared to ordinary silicon one $(\leq 10 \mathrm{~nm})$ can lead to lower lateral resolution. However, this effect is found to be not critical for the performed measurements. Scanning electron microscopy (SEM) images are obtained by a Zeiss 1540 XB field emis- 


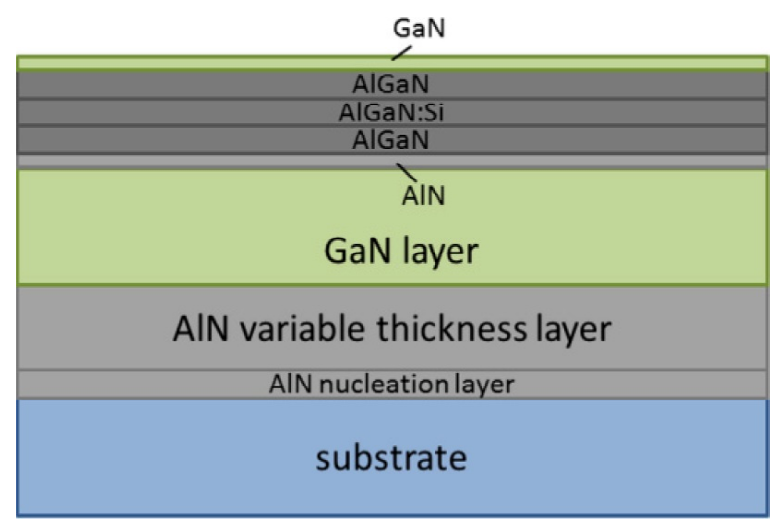

Fig. 1. Schematic picture of the grown HEMT structures on sapphire substrates. It includes thin (a few tens of $\mathrm{nm}$ ) AIN nucleation layer, AIN layer of variable thickness, GaN layer of 800-950 nm in thickness, about $1 \mathrm{~nm}$ thick AIN capping layer, a stack of AlGaN layers with thicknesses of a few nm each, where the middle layer is doped with $\mathrm{Si}$, and about $3 \mathrm{~nm}$ thick GaN capping layer.

sion microscope. AFM and SEM studies are carried out on the as grown as well as on etched samples to estimate dislocation density. The etching is performed by melting $\mathrm{KOH}$ pellets at $220^{\circ} \mathrm{C}$ on a hot plate inside an alumina crucible. The samples are dipped in the melt for 9 minutes and then quenched in water at room temperature.

Rutherford back scattering (RBS) experiments are performed using $2 \mathrm{MeV} \mathrm{He}^{+}$ions. A Si(Li) detector with $30 \mathrm{eV}$ resolution is used to collect the ions at a backscattering angle of $165^{\circ}$. The channels are calibrated using $\mathrm{Si}$ falling edge and Au falling edge. The SIMNRA software package is used to simulate the results to quantify the thickness and composition of the layers. Secondary ion mass spectrometry (SIMS) is carried out using a Hiden Analytical SIMS Workstation. Sputtering is done with oxygen ions at $5 \mathrm{keV}$ energy and a current of $200 \mathrm{nA}$. Charge neutralization is performed by an electron flood gun with $500 \mathrm{eV}$ energy and a current of $750 \mu \mathrm{A}$. Secondary ions are detected using a quadrupole analyzer.

$\mathrm{X}$-ray diffraction $(\mathrm{XRD})$ measurements are carried out using a PANalytical Empyrean and utilizing copper $\mathrm{K} \alpha_{1,2}$ lines. The samples are rotated around the $c$-axis (perpendicular to basal plane of AIGaN/ GaN/AIN) in order to average contributions from inplane directions.

Optical properties of the heterostructures are studied by photoluminescence $(\mathrm{PL})$ spectroscopy at room and low temperatures. In the room temperature PL measurements, the samples are ex- cited by an unfocused Ar-ion laser with a wavelength corresponding to $3.53 \mathrm{eV}$. The laser beam comes under $45^{\circ}$ angle with the normal to the surface and the PL emission is collected along the normal, thus, avoiding the direct reflection of the beam into the detection path. Low temperature PL measurements are carried out using a $4.43 \mathrm{eV}$ photodiode with focused excitation beam coming through a quartz window in a flow cryostat cooled by liquid $\mathrm{He}$ to a temperature of $37 \mathrm{~K}$. To obtain high resolution spectra of the exciton bands, the temperature is lowered to $21 \mathrm{~K}$. In all measurements, the excitation flux is kept at a low values to avoid saturation effects. The $\mathrm{PL}$ signal is analyzed by an electron multiplying CCD mounted on a fiber-coupled spectrometer using both 150 lines $/ \mathrm{mm}$ and 1200 lines/mm gratings. The recorded spectra have not been corrected for the wavelength dependent quantum efficiency of the detector.

Concentration and mobility of charge carriers are obtained from standard Hall measurements performed in the van der Pauw configuration using a home-made setup utilizing a four point probe station, a permanent disc magnet and a translation stage for varying the position of the magnet with respect to the sample. The contacts are deposited by indium solder on top of the heterostructures. The $\mathrm{I}-\mathrm{V}$ characteristics of the contacts are measured to verify their ohmic nature.

\section{RESULTS AND DISCUSSION}

The surface morphology of the different samples looks very similar. As examples, AFM images for samples $A$ and $C$ are presented in Figs. $2 a$ and $2 b$, respectively, illustrating surface with terraces. Surface roughness varies slightly from sample to sample yielding root mean square (RMS) values between 1.7 and $2.4 \mathrm{~nm}$ on areas of $5 \times 5 \mu \mathrm{m}$. However, there is a tendency that average height variations are a bit wider for samples $B$ and $C$ (those with thinner AIN buffer layer) compared to samples $A$ and $D$. Samples $B$ and $C$ also show more nanometer-size protrusions as those that can be seen in Fig. $2 b$. Imaging of smaller areas (see Fig. 2c) clearly shows a number of pits located at joint points of terraces, thus, allowing to associate the pits with V-shaped defects decorating TDs. These dislocations are caused by the compressive stresses in the AIN layer due to the lattice mismatch with the sapphire substrate. During epitaxial growth the dislocations continue through the interfaces into GaN and further into the top layers of the heterostructures [16]. The surface density of V-shaped defects is found to 

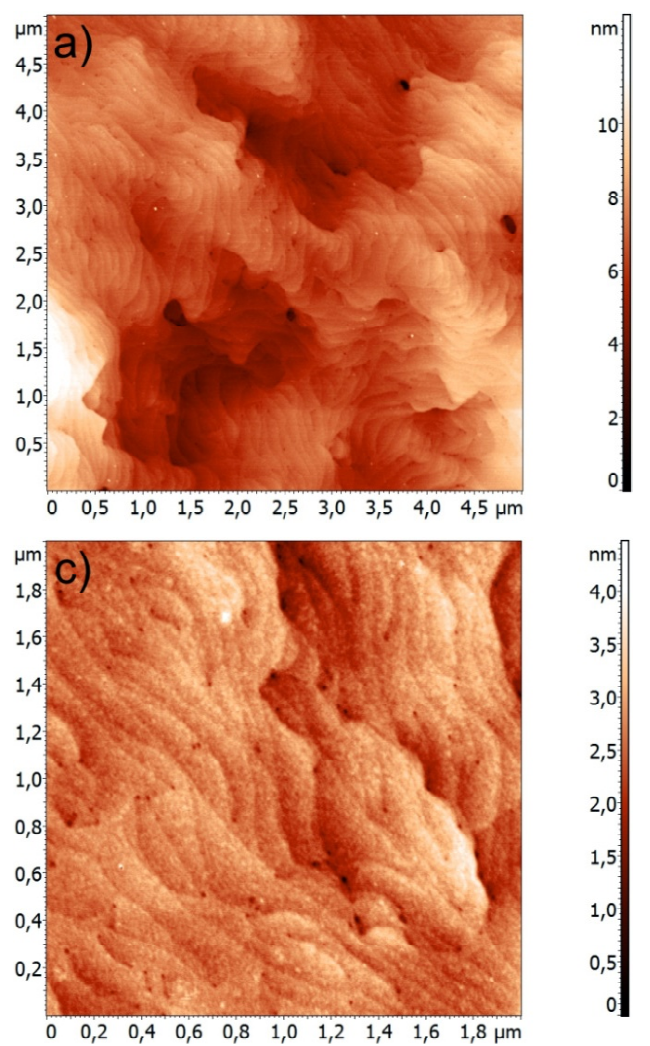

$n m$
4,0
3,5
3,0
2,5
2,0
1,5
1,0
0,5
0

be approximately $2 \times 10^{9} \mathrm{~cm}^{-2}$ for samples of type $A$ and $D$, while $4 \times 10^{9} \mathrm{~cm}^{-2}$ for samples of type $B$ and C. Thus, one can conclude that dislocation densities are approximately two times lower for the samples with thicker AIN layers. This is caused by reduced crystal misorientation and stress as a result of the increased thickness, which is a well-known phenomenon [17]. The TDs can be either of a-type with Burgers vector $b=1 / 3<112 \overline{0}>$, $a+c$-type $b=$ $1 / 3<112 \overline{3}>$ or $c$-type $b=<0001>$, typically referred to as edge, mixed or screw TDs, respectively [10]. However, we did not study the exact types of dislocations in our case.

Chemical etching is known to help in better resolving a dislocation termination at the surface. SEM images for all types of samples after the etching are shown in Fig. 3. One can see that the etched pits have hexagonal shapes following the GaN polytype (wurtzite structure). Every pit should correspond to an individual TD [18]. Samples B and C demonstrate higher pit densities compared to $A$ and D. However, in all cases these densities are about 2 orders of magnitude lower compared to those found by AFM for V-shaped defects. Using AFM for the etched samples clarifies the difference. In Fig. 4a, one can see an image of one of the samples after the etching. Indeed, only a few hexagonal pits per area of $4 \times 4 \mu \mathrm{m}$ can be observed that correlates with the SEM findings. However, if one looks into im-

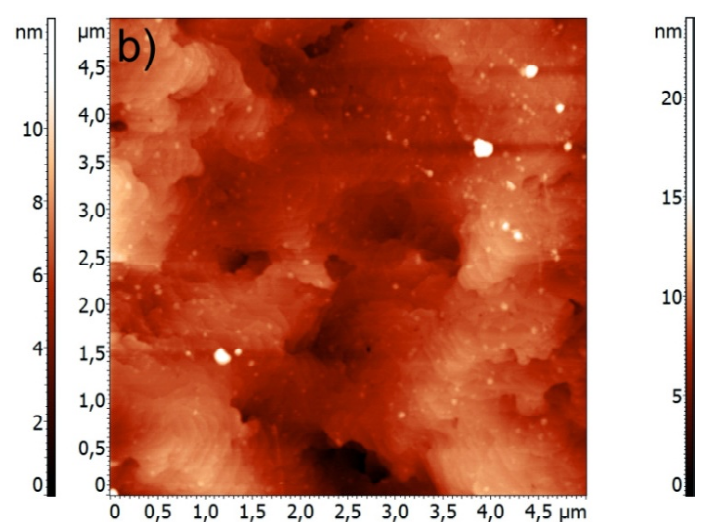

Fig. 2. AFM topography images of samples (a) A and (b) $C$ on the scale $5 \times 5 \mu m^{2}$ as well as (c) $A$ on the scale $2 \times 2 \mu \mathrm{m}^{2}$ clearly visualizing $V$-defects as small dark spots.

ages obtained on smaller areas (with higher resolution), it is possible to see smaller in diameter pits which are not developed into hexagonal structures. They are clearly identified in phase images. To illustrate the correlation a few corresponding pits are marked by arrows in the topography image (dark spots in Fig. 4b) and phase image (bright spots in Fig. 4c). Thus, it can be concluded that the etching in our case does not develop all V-defects into hexagonal pits. This can be related to the fact that different types of TD have different etching efficiencies. Therefore, in the SEM images only a limited number of dislocations are visible while AFM allows finding the absolute majority of them even without etching [19].

Composition and thickness of the grown structures are studied using SIMS and RBS. The advantage of SIMS is in high resolution of concentration of elements. However, it is impossible to justify thickness of analyzed layers without reference samples. Although RBS can also give the composition of a material, its resolution, especially for light chemical elements such as nitrogen in our case, is low. However, one can obtain thickness of the analyzed films very precisely.

SIMS spectra of samples $A$ and $D$ obtained at identical sputtering conditions are presented in Figs. $5 \mathrm{a}$ and $5 \mathrm{~b}$, respectively. Concentration fluctuations in the beginning of sputtering are related to $\mathrm{nm}$-thick 
top barrier layers (see Fig. 1). However, we are mainly interested in the composition of GaN and buffer AIN layers. As can be seen, concentrations of $\mathrm{Ga}$ and $\mathrm{N}$ are approximately the same in the top GaN layer in both panels. Al is present in the GaN film at trace amounts and one can see a sharp change in Al concentration at the AIN interface. Due to the difference in ionization probabilities of Ga and N in SIMS, one cannot judge about stoichiometry of the syn-
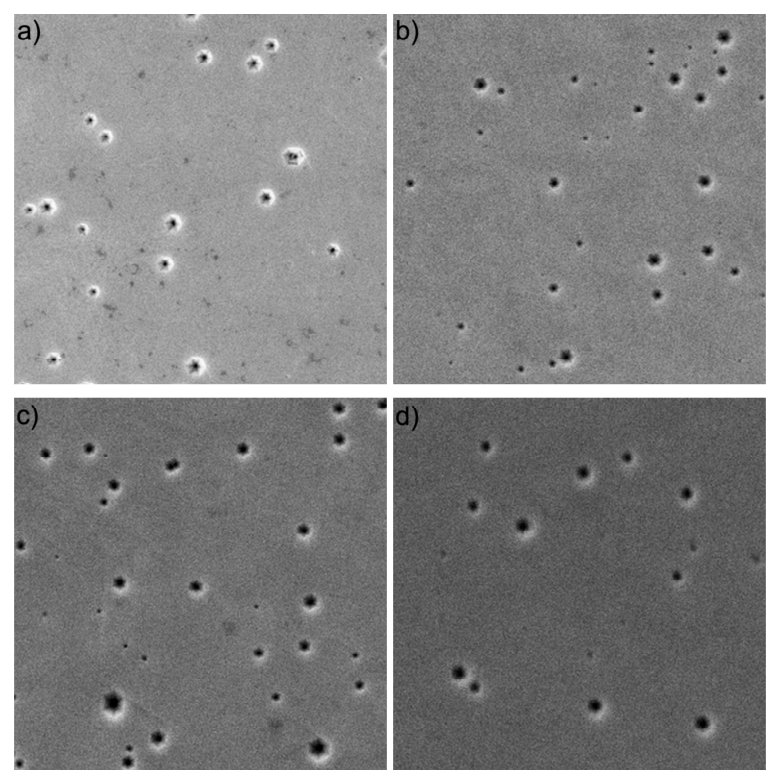

Fig. 3. SEM images of samples (a) A, (b) B, (c) C and (d) D. Frames of all images are $10 \times 10 \mu \mathrm{m}^{2}$.
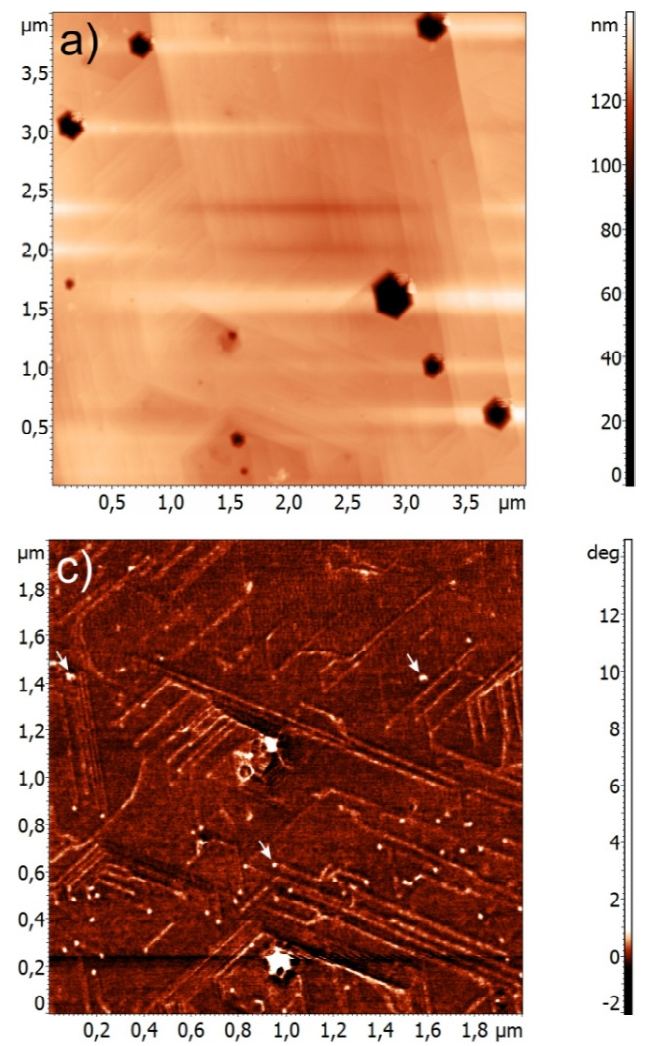

thesized GaN. These conclusions as well as estimations of layer thickness can be drawn from the RBS measurements and simulations of the obtained spectra. An example of an experimental RBS spectrum and simulated curve is given in Fig. 6 for sample D. From RBS, the thickness of stoichiometric $\mathrm{GaN}$ is found to be ca. 900 and $950 \mathrm{~nm}$ for samples $A$ and $D$, respectively. This is in good correlation with the SIMS scans showing slightly longer sputtering time for sample $D$ compared to $A$.

In the SIMS scans (Figs. $5 a$ and $5 b$ ), the GaN film is followed by buffer AIN layer. For sample $A$, it is grown under constant flows of TMAl and $\mathrm{NH}_{3}$ while for sample $\mathrm{D}$ the flow of $\mathrm{NH}_{3}$ is gradually decreased with time (see Experimental for details). In both panels (a) and (b) one can see flat dependences of $\mathrm{Al}$ and $\mathrm{N}$ concentrations on the sputtering time. Thus, it does not seem that the gradual decrease of ammonia flow during the growth of sample $D$ affects the composition of the epitaxial layer. However, it can be observed in Figs. $5 \mathrm{a}$ and $5 \mathrm{~b}$ that the $\mathrm{Ga}$ concentration does not drop abruptly at GaN/AIN interface. RBS data also confirm formation of nonsharp interface with gradual decrease of Ga from 50 to approximately 10 at.\% over a thickness of ca. $210 \mathrm{~nm}$ and $90 \mathrm{~nm}$ in samples $A$ and $D$, respectively. Thus, both SIMS and RBS measurements show evidence of considerable diffusion of Ga into AIN. The total thickness of the AIN film (including
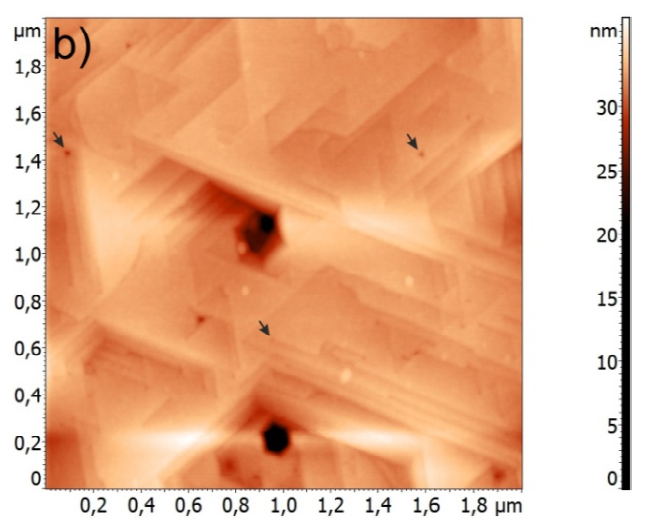

Fig. 4. AFM images of sample $C$. Topography on scale (a) $5 \times 5 \mu m^{2}$ and (b) $2 \times 2 \mu m^{2}$ as well as (c) phase images of the same area as in panel (b). Arrows in (b) and (c) show a few identical defect points. 

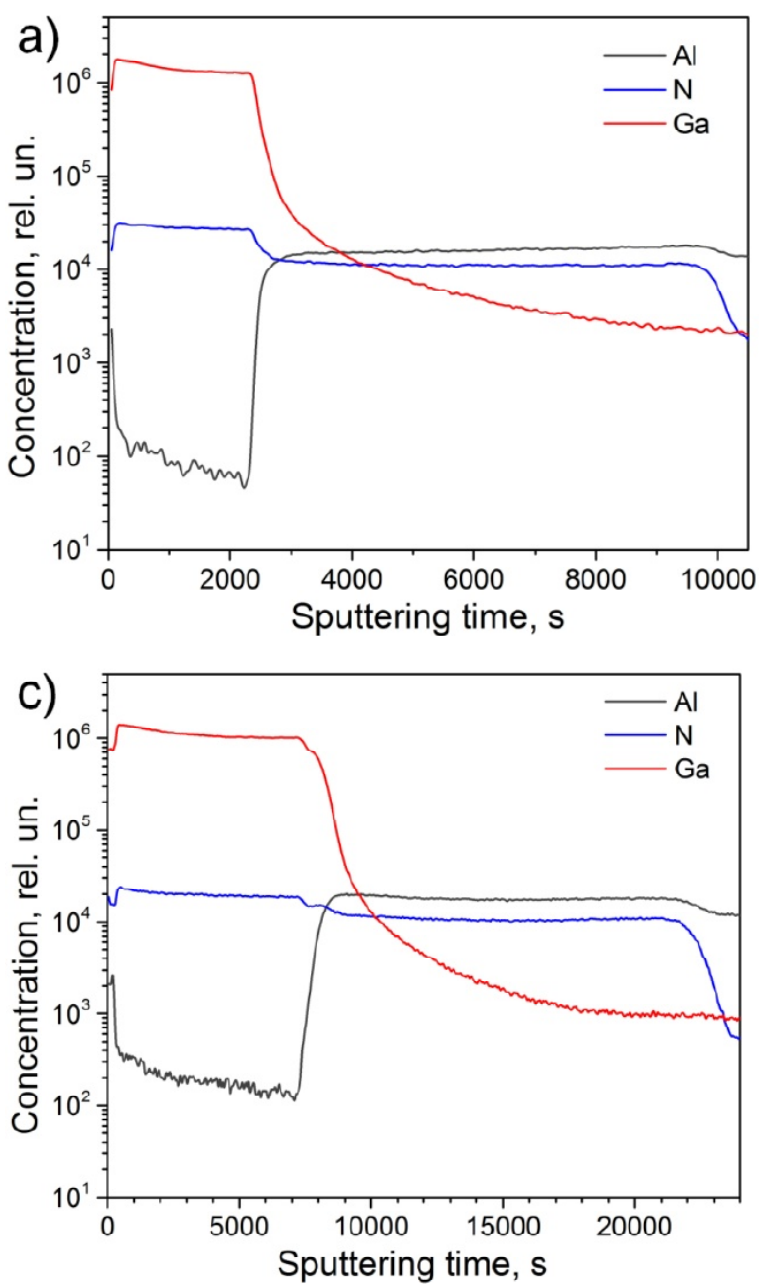

the layers with diffused $\mathrm{Ga}$ ) is found to be ca. 700 and $800 \mathrm{~nm}$ in samples $A$ and $D$, respectively, that is in good agreement with the sputtering times presented in Fig. $5 \mathrm{a}$ and $5 \mathrm{~b}$ for this layer (longer time for sample D).

As SIMS measurements of samples B and C show very similar concentration profiles, only the profile for sample $\mathrm{C}$ is shown in Fig. 5c. This profile is obtained for scan areas different in size compared to those in panels (a) and (b). This explains the difference in the sputtering times. Similar to the previous samples, the spectrum shows flat concentration dependences of $\mathrm{Ga}$ and $\mathrm{N}$ in the GaN film and RBS yields the thickness of stoichiometric GaN of about $800 \mathrm{~nm}$. This film is followed by approximately $150 \mathrm{~nm}$ thick layer of AIN characterized by the presence of a significant amount of $\mathrm{Ga}$ with decreasing concentration from 50 to 10 at.\%. Then, the $\mathrm{Ga}$ amount in the AIN layer quickly drops down similar to the cases of samples A and D. The total thickness of AIN (including layers with diffused $\mathrm{Ga}$ ) is estimated from RBS to be around $500 \mathrm{~nm}$. Thus, this layer is considerably thinner in samples $B$ and $C$ compared to $A$ and $D$, as expected from the growth

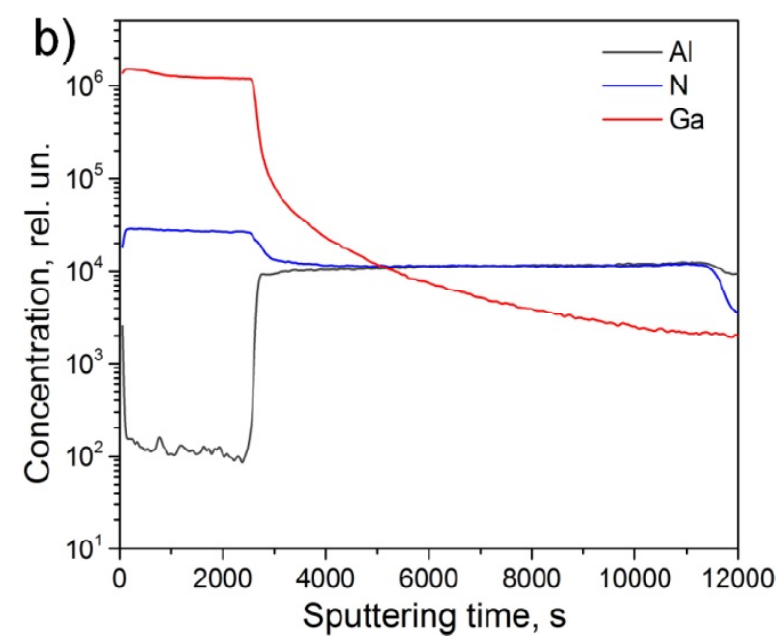

Fig. 5. Concentration profiles of $\mathrm{Al}, \mathrm{Ga}$, and $\mathrm{N}$ as a function of sputtering time obtained by SIMS for samples (a) A, (b) D and (c) C.

conditions. The change of $\mathrm{Al}$ concentration at the GaN/AIN interface in Fig. $5 \mathrm{c}$ is not so sharp compared to the dependences in Figs. $5 \mathrm{a}$ and $5 \mathrm{~b}$. One can also see stepwise changes in $\mathrm{Ga}$ and $\mathrm{N}$ amounts at the interface. All these findings indicate less sharp interfaces with increased interdiffusion of elements in samples $B$ and $C$ compared to samples $A$ and $D$.

XRD spectra for all 4 types of samples are presented in Fig. 7. Using the literature data $[20,21]$, the peaks are associated with $\mathrm{GaN}(0002), \operatorname{AIN}(0002)$, and $A I G a N(0002)$. GaN peaks for samples $A$ and $D$ have about the same intensities indicating similar thickness and crystalline quality of the layers which is in good agreement with the AFM and RBS data. The same tendency is found for samples $B$ and $C$, but intensities of these peaks are a little lower compared to those obtained on samples $A$ and $D$ which also correlates well with the RBS measurements showing thinner GaN layer for samples B and C. Intensities of AIN peaks once again demonstrate lower thickness of this layer when comparing samples $B$ and $C$ with $A$ and $D$. The intensity of the shoulder associated with $\mathrm{AlGaN}$ is low and it is about 


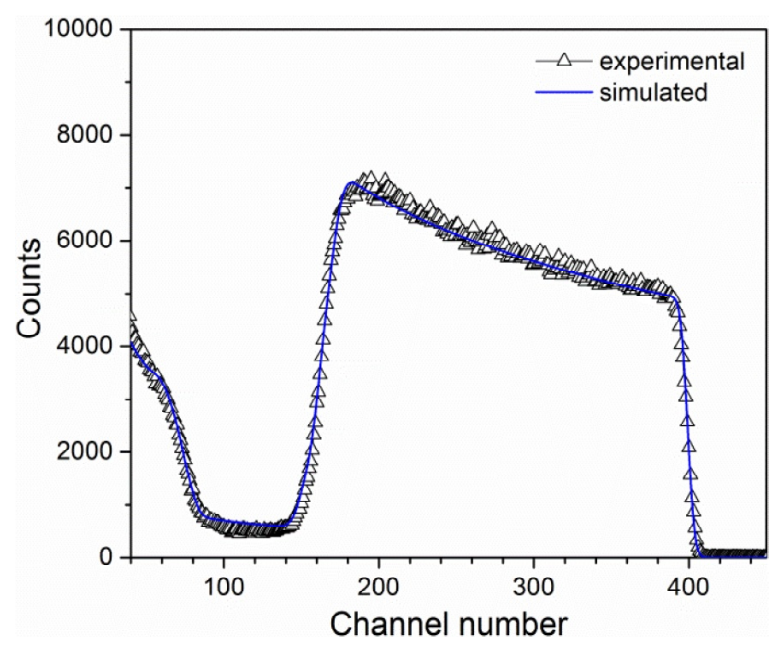

Fig. 6. Experimental RBS spectrum for sample $C$ and corresponding simulated curve used for the calculation of atomic concentration of elements as a function of depth.

the same for all cases because it corresponds to the stack of nm-thick top layers which are grown under exactly the same conditions for all samples. The XRD results very well support the data obtained by SIMS and RBS, thus, making the conclusion better grounded.

Room temperature PL spectra are recorded such that the total integrated signal for each sample can be directly compared. The excitation energy of 3.53 $\mathrm{eV}$ allows probing mainly the GaN layer since it renders the top AIGaN and AIN layer transparent. Penetration depth for this photon energy in GaN is estimated to be around $100 \mathrm{~nm}$ [22]. All spectra (see Fig. 8) show a sharp near band edge (NBE) luminescence peak at $3.430 \mathrm{eV}$. The next sharp peak at lower energy of $3.343 \mathrm{eV}$ is most likely the

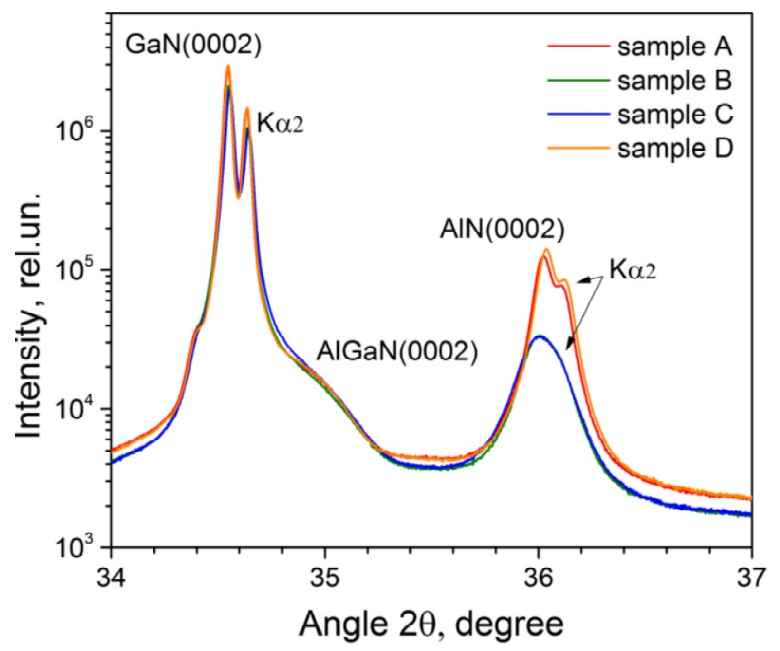

Fig. 7. XRD spectra obtained for samples A, B, C, and $\mathrm{D}$. 2nd order Raman line of the $A_{1}$ longitudinal optical (LO) mode in GaN having high intensity due to the resonant excitation energy used and because the $\mathrm{GaN}$ is transparent for this photon energy. In the lower energy interval, a characteristic band corresponding to yellow luminescent $(\mathrm{YL})$ can be observed. This band is related to a deep acceptor impurity present in almost any wurtzite GaN sample. The nature of this band has been extensively studied and assigned to carbon and a carbon-oxygen complexes substituting nitrogen in the lattice $[23,24]$. The observed intensity ripples of the YL band are most probably caused by multiple reflections of the generated luminescence in the whole GaN stack. Samples B and C show about the same total PL intensity suggesting similar quantum efficiencies of the GaN layers. Samples A and D demonstrate approximately 2.5 and 5 times higher PL intensities, respectively, compared to samples $B$ and $C$. The ratios of $Y L$ to NBE are very close for all samples while being slightly higher for samples $A$ and $D$, which can be explained by the defects responsible for the YL band having a higher quantum yield compared to the recombination of free carriers [15]. The increase in total quantum efficiency for sample $A$ and $D$ compared to sample $B$ and $C$ can be an indication of either reduction in the amount of non-radiative recombinations or lower TD density. TDs may act as non-radiative recombination sites expected to degrade optical and electronic properties of the material [25].

To distinguish between these two mechanisms affecting the optical properties, the PL spectra are measured at cryogenic temperature, see Fig. 9. Lowtemperature PL measurements typically allow to visualize the contribution of defects affecting the

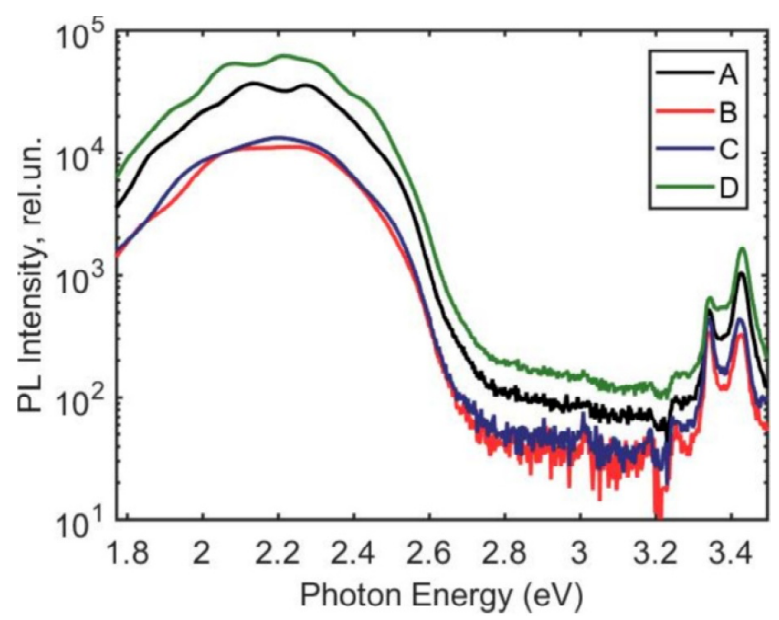

Fig. 8. Room temperature photoluminescence spectra of the heterostructures. 


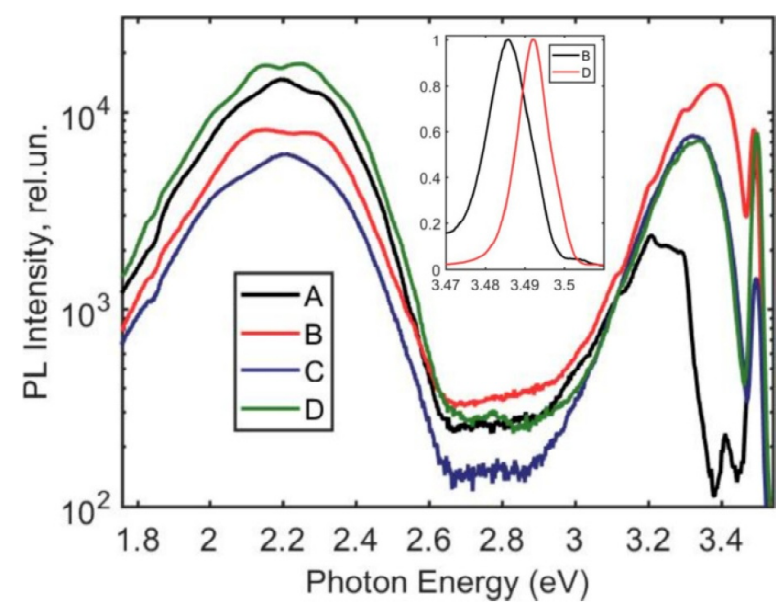

Fig. 9. Low-temperature photoluminescence spectra of the heterostructures. Insert show high resolution exciton bands (see text for details).

luminescent transitions as well as the free exciton transitions which dominate over the donor and acceptor bound exciton transitions having low activation energies. The spectra show the $Y L$ bands very similar to those observed at room temperature. Absence of additional to YL broad bands at low-temperature measurements indicates that the growth is free from other than carbon or carbon-oxide impurities incorporated as deep carrier traps in the lattice which are sometimes seen in low-energy interval of PL spectra of GaN films synthesized by MOVPE [15]. At energies above $3 \mathrm{eV}$, an unidentified luminescence band with varying intensity is observed. This band is difficult to study and interpret due to its overlap with an ultraviolet luminescence band at $3.29 \mathrm{eV}$ which is quite typical for GaN samples and related to presence of $\mathrm{Mg}$ [15]. This band can be followed by a few LO phonon replicas redshifted with respect to the main transition line. One can clearly see three LO phonon replicas for sample B. They are also nicely pronounced in the spectrum of sample $D$ suggesting a long range high crystalline quality of GaN [15].

However, the most interesting spectral feature in this case is an exciton band at around $3.49 \mathrm{eV}$ [26]. Measurements carried out on a commercial free-standing GaN crystal (spectra are not included here) show that this band is actually composed of 3 distinct exciton lines: two of which are the free exciton transitions and one belongs to a donor bound exciton transition. The measurements on the heterostructures do not provide this splitting but, nevertheless, show bands that are sharp enough to be compared for different samples as can be seen in the insert to Fig. 9. Sample D shows the blue shift of the maximum for $0.006 \mathrm{eV}$ and lower full width at half maximum (FWHM) value compared to sample B. The observed shift can be interpreted as a difference in strain in the GaN layers of these two samples, thus, indicating difference in TD and point defect densities affecting the crystalline quality. It is also known that poor crystalline quality of GaN increases the exciton linewidth [27]. Hence, comparing the bands one can conclude about lower TD and defect density in sample $\mathrm{D}$ compared to $\mathrm{B}$. This optically-based conclusion is in very good agreement with those drawn from the structural and compositional studies of the synthesized heterostructures.

The results of electrical measurements are presented in Table 1. They show quite similar values of the charge carrier concentration for all samples which are on the order of $1 \times 10^{13} \mathrm{~cm}^{-2}$ and are comparable to the state-of-the-art values [28-30]. The highest mobility values $\mu$, found for samples $A$ and $D$, are, however, lower compared to those published elsewhere (approximately between $1400-2185 \mathrm{~cm}^{2} / \mathrm{V} \cdot \mathrm{s}$ ) [28-30]. The main reason for this is relatively high TD density for our samples which reduces the Hall mobility as found earlier in [14]. The lowest TD density of about $2 \times 10^{9} \mathrm{~cm}^{-2}$ for samples $A$ and $D$ yields $\mu=1130-1150 \mathrm{~cm}^{2} / \mathrm{V} \cdot \mathrm{s}$. An increase of TD density by a factor of two in samples $B$ and $C$ leads to decrease of the mobility also roughly two times. The higher dislocation density in these heterostructures is most probably related to thinner AIN buffer layers which are not able to accommodate the stresses developed due to the lattice mismatch. As shown elsewhere, the dislocation density is inversely proportional to the layer thickness; with its increase there is a higher probability for neighboring TDs to annihilate and fuse, thus, decreasing the total dislocation density [31]. Hence, it can be concluded that further optimization of AIN growth parameters is required in order to achieve lower dislocation densities in AIGaN/GaN structures providing higher electron mobility.

Table 1. Samples resistance, carrier concentration and mobility measured by Hall method in van der Pauw configuration.

\begin{tabular}{llll}
\hline Sample & $\begin{array}{l}\mathrm{R}_{\mathrm{s}} \\
(\Omega / \mathrm{sq} .)\end{array}$ & $\begin{array}{l}n \\
\left(\mathrm{~cm}^{-2}\right)\end{array}$ & $\begin{array}{l}\mu \\
\left(\mathrm{cm}^{2} \mathrm{~V}^{-1} \mathrm{~s}^{-1}\right)\end{array}$ \\
\hline $\mathrm{A}$ & 541 & $1.02 \times 10^{13}$ & 1130 \\
$\mathrm{~B}$ & 1030 & $0.97 \times 10^{13}$ & 622 \\
$\mathrm{C}$ & 800 & $1.07 \times 10^{13}$ & 730 \\
$\mathrm{D}$ & 474 & $1.14 \times 10^{13}$ & 1150 \\
\hline
\end{tabular}




\section{CONCLUSIONS}

In the current work, AIGaN/GaN heterolayers are formed by MOVPE and the structure, composition as well as photoluminescence and electrical parameters of the samples are studied with focus on the investigation of how the conditions of AIN buffer growth affect the quality of the top III-N layers and the formation of a 2DEG. Using SEM and AFM measurements the density of $\mathrm{V}$-defects is found and associated with the density of TD which is calculated to vary between (2-4) $\times 10^{9} \mathrm{~cm}^{-2}$ depending on the synthesis parameters. These values are higher than the typical state-of-the-art literature data indicating moderate quality of the grown heterostructures with the sheet resistance values for our samples to be 1.5-2 times higher compared to the best reported results. It is worth mentioning that the high dislocation density mostly affects the electron mobility while only moderately impacts the charge carrier concentrations. In order to resolve the reasons for a high TD density, a thorough study of structure and composition has been carried out using RBS, SIMS and XRD. It is found that III-N layers are stoichiometric in composition and thickness of the films corresponds to the expectations from the growth conditions. Synthesis of the heterostructures on thicker AIN buffer shows fewer dislocations and, thus, lower electrical resistance. The attempt of gradual decrease of ammonia flow during the growth of the buffer layer does not seem to affect the composition of the epitaxial layer and the crystalline quality of the top GaN film. Thus, this approach can be excluded from further optimisation. One of the interesting observed phenomena is considerable diffusion of Ga from the top GaN film into the buffer AIN layer. The origin of this effect is not clear and it requires special investigations in order to see how it affects the crystalline quality of the GaN layer. The photoluminescence study indicates the presence of carbon-related defects in GaN leading to typical yellow band in the spectra. Analysis of the exciton band in low-temperature optical spectra supports very well the conclusions of structural analysis on lower TD densities in GaN grown on thicker AIN buffer layers. The obtained results are an important step for further optimization of the MOVPE growth, in particular, through the adjustment of AIN synthesis towards formation of HEMT structures on sapphire and transfer of technologies to Si substrates.

\section{ACKNOWLEDGEMENTS}

The authors acknowledge financial support from the Innovation Fund Denmark within the project Semi- conductor Materials for Power Electronics (SEMPEL).

\section{REFERENCES}

[1] O. Ambacher, J. Smart, J. R. Shealy, N. G. Weimann, K. Chu, M. Murphy, W. J. Schaff, L. F. Eastman, R. Dimitrov, L. Wittmer, M. Stutzmann, W. Rieger and J. Hilsenbeck // J. Appl. Phys. 85 (1999) 3222.

[2] J. Millan, P. Godignon, X. Perpina, A. PerezTomas and J. Rebollo // IEEE Transact. Power Electron. 29 (2014) 2155.

[3] P. Gibart // Rep. Prog. Phys. 67 (2004) 667.

[4] S. Keller, H. Li, M. Laurent, Y. Hu, N. Pfaff, J. Lu, D.F. Brown, N.A. Fichtenbaum, J.S. Speck and S.P DenBaars // Semicond. Sci. Technol. 29 (2014) 113001.

[5] S.A. Kukushkin, A.V. Osipov, V.N. Bessolov, B.K. Medvedev, V.K. Nevolin and K.A. Tcarik // Rev. Adv. Mater. Sci. 17 (2008) 1.

[6] L. Zhang, K.H. Lee, I.M. Riko, C.-C. Huang, A. Kadir, K.E. Lee, S.J. Chua and E.A. Fitzgerald // Semicond. Sci. Technol. 32 (2017) 065001.

[7] S.A. Kukushkin, A.V. Osipov, V.N. Bessolov, E.V. Konenkova and V.N. Panteleev // Phys. Sol. State 59 (2017) 674.

[8] M.M. Rozhavskaya, S.A. Kukushkin, A.V. Osipov, A.V. Myasoedov, S.I. Troshkov, L.M. Sorokin, R.S. Telyatnik, R.R. Juluri, K. Pedersen and V.N. Popok // Phys. Stat. Sol. A 214 (2017) 1700190.

[9] J. Cheng, X. Yang, L. Sang, L. Guo, J. Zhang, J. Wang, C. He, L. Zhang, M. Wang, F. Xu, N. Tang, Z. Qin, X. Wang and B. Shen // Sci. Rep. 6 (2016) 23020.

[10] M.A. Moram, C.S. Ghedia, D.V.S. Rao, J.S. Barnard, Y. Zhang, M.J. Kappers and C.J. Humphreys // J. Appl. Phys. 106 (2009) 073513.

[11] S.W. Kaun, M.H. Wong, U.K. Mishra and J.S. Speck // Appl. Phys. Lett. 100 (2012) 262102.

[12] I.P. Smorchkova, L. Chen, T. Mates, L. Shen, S. Heikman, B. Moran, S. Keller, S. P. DenBaars, J. S. Speck and U. K. Mishra // J. Appl. Phys. 90 (2001) 5196.

[13] A. Asgari, S. Babanejad and L. Faraone // J. Appl. Phys. 110 (2011) 113713.

[14] S. W. Kaun, B. Mazumder, M. N. Fireman, E. C. H. Kyle, U. K. Mishra and J. S. Speck // Semicond. Sci. Technol. 30 (2015) 055010. 
[15] M. A. Reshchikov and H. Morkoç // J. Appl. Phys. 97 (2005) 061301.

[16] B. Heying, E. J. Tarsa, C. R. Elsass, P. Fini, S. P. DenBaars and J. S. Speck // J. Appl. Phys. 85 (1999) 6470.

[17] D. Zhu, D.J. Wallis and C.J. Humphreys // Rep. Prog. Phys. 76 (2013) 106501.

[18] T. Hino, S. Tomiya, T. Miyajima, K. Yanashima, Hashimoto and M. Ikeda // Appl. Phys. Lett. 76 (2000) 3421.

[19] A. Pandey, B.S. Yadav, D.V.S. Rao, D. Kaur and A.K. Kapoor // Appl. Phys. A 122 (2016) 614.

[20] H.-Y. Shih, M. Shiojiri, C.-H. Chen, S.-F. Yu, C.-T. Ko, J.-R. Yang, R.-M. Lin and M.-J. Chen // Sci. Rep. 5 (2015) 13671.

[21] H.-P. Lee, J. Perozef, L.D. Rosario and C. Bayram // Sci. Rep. 6 (2016) 37588.

[22] B. Ozden, M. P. Khanal, S. Youn, V. Mirkhani, K. Yapabandara, M. Park, M. Zhao, H. Liang, P. K. Kandaswamy, and Y. N. Saripallib // ECS J. Sol. State Sci. Technol. 5 (2016) P3206.
[23] J.L. Lyons, A. Janotti and C. G. Van de Walle // Appl. Phys. Lett. 97 (2010) 152108.

[24] M.A. Reshchikov, D.O. Demchenko, A. Usikov, H. Helava and Yu. Makarov // Phys. Rev. B 90 (2014) 235203.

[25] M. Albrecht, J.L. Weyher, B. Lucznik, I. Grzegory and S. Porowski // Appl. Phys. Lett. 92 (2008) 231909.

[26] D.C. Reynolds, J. Hoelscher, C.W. Litton and T.C. Collins // J. Appl. Phys. 92 (2002) 5596.

[27] M. A. Reshchikov // Phys. Stat. Sol. C 8 (2011) 2136.

[28] M.J. Manfra, N.G. Weimann, J.W.P. Hsu, L.N. Pfeiffer, K.W. West and S.N.G. Chu // Appl. Phys. Lett. 81 (2002) 1456.

[29] X. Wang, C. Wang, G. Hu, H. Xiao, C. Fang, J. Wang, J. Ran, J. Li, J. Li and Z. Wang // J. Cryst. Growth 298 (2007) 791.

[30] W.-C. Huang, C.-M. Chu, Y.-Y. Wong, K.-W. Chen, Y.-K. Lin, C.-H. Wu, W.-I. Lee and E.Y. Chang // Mater. Sci. Semicond. Proc. 45 (2016) 1.

[31] A.E. Romanov, W. Pompe, G. Beltz and J.S. Speck // Phys. Stat. Sol. (a) 198 (1996) 599. 